\title{
A Tale of Two Inspection Methods: Comparing an eHealth Literacy and User Experience Checklist with Heuristic Evaluation
}

\author{
Helen MONKMAN ${ }^{\mathrm{a}, 1}$ and Janessa GRIFFITH ${ }^{\mathrm{b}, \mathrm{c}}$ \\ ${ }^{a}$ School of Health Information Science, University of Victoria, Canada \\ binstitute for Health System Solutions and Virtual Care, \\ Women's College Hospital, Toronto, Canada \\ ${ }^{c}$ Faculty of Health Sciences, Douglas College, Coquitlam, Canada
}

\begin{abstract}
Adhering to user experience (UX) and eHealth literacy principles when developing consumer health information systems (HISs) can not only improve a user's experience but can also have implications on patient safety. Methods exist to explore these dimensions independently, but few methods are available for evaluating consumer (i.e., citizen) health information systems for their adherence to usability and eHealth literacy design principles simultaneously. In this paper, we compared two inspection (i.e., expert review) tools and identified the strengths and weaknesses of each. The findings from this comparison can assist researchers, consumer health information system developers, and evaluators choosing between the two alternatives. Moreover, our comparison revealed the shortcomings in both tools and the need for a novel, purpose-built tool that is more comprehensive than either of the existing tools that assess UX and eHealth literacy and more adequately address design guidelines for the mobile environment.
\end{abstract}

Keywords. Consumer Health Informatics, Usability, User Experience, Health Literacy, eHealth Literacy, Digital Health

\section{Introduction}

Citizens (i.e., lay people, health consumers, patients) are increasingly turning to digital resources and tools referred to as consumer health information systems (HISs) (e.g., websites, mobile applications (apps), personal health records) to support their health. For example, in a 2014 report, approximately $59 \%$ of Europeans sought health information online in the previous year [1]. As an index of current demand, approximately 200 new mobile health apps are added to app stores daily [2]. Some organizations offer curated sets of accredited consumer health apps (e.g., the NHS app library), although many health apps and websites are available to citizens without being subject to any oversight. Consumer HISs can be informational, educational, and provide tools to help manage, monitor, and prevent illness. Many argue that the adoption and success of health

${ }^{1}$ Corresponding Author, Helen Monkman, Health Information Science, University of Victoria, PO Box 1700 STN CSC, Victoria BC,V8W 2Y2, Canada, E-mail: monkman@uvic.ca. 
information systems hinge on them being usable (e.g., [3]). Usability is "the effectiveness, efficiency and satisfaction with which specified users achieve specified goals in particular environments" [4]. Over time, the emphasis on satisfaction increased and user experience (UX) became a more popular term. Usability is a fundamental component of UX, but UX extends beyond usability and is defined as "a person's perceptions and responses that result from the use or anticipated use of a product, system or service" [4].

Health information systems are often complex the reflect the complexity of health care itself. However, consumer HISs face an additional challenge: they are meant to be used by people who are not health experts and often have limited or no related health knowledge. eHealth literacy is "the ability to seek, find, understand, and appraise health information from electronic sources and apply the knowledge gained to addressing or solving a health problem" and these skills are essential for engaging with consumer HISs [5]. Therefore, in the context of consumer HISs, eHealth Literacy and usability (or more broadly UX) cannot be disentangled. That is, if a system is difficult or unpleasant to use, people will not use it or find what they are looking for and if the content is not well communicated, people will not understand it or could misunderstand it [6]. Issues with eHealth literacy have been identified inadvertently [7] and purposefully in usability evaluations [8]. Therefore, failing to address both UX and eHealth literacy in consumer HISs creates risks of limited adoption or worse, poor decision making as a result of misunderstanding. Although these two constructs can be investigated independently, it is more efficient to evaluate them in conjunction. As such, practical, easy to apply, evidence-based evaluation frameworks for eHealth literacy and UX to assess consumer HISs are deeded, yet few tools evaluate both of these dimensions simultaneously.

This paper compares two evidence-based tools that can be used to assess UX and eHealth literacy in conjunction: The Health Literacy Online (HLO) Checklist [9] and Evidence-Based Heuristics for Usability and eHealth Literacy [10]. Both tools are inspection methods (i.e., expert reviews). Inspection methods are valuable, informal, "first lines of defense" against usability problems because they require relatively limited human, financial, and time-related resources [11]. However, inspection methods cannot be substituted for usability tests with representative users attempting tasks in real environments because the two approaches often reveal different usability issues [11].

\section{Health Literacy Online}

Recognizing that eHealth literacy and usability are inextricable components of consumer HISs and in an effort to support the design of health websites for the public, the Department of Health and Human Services in the United States developed a resource called Health Literacy Online: A Guide to Writing and Designing Easy-to-Use Health Web Sites (HLO) [12]. Originally launched in 2010, the evidence-based resource was available as a website or a PDF report. In its second iteration, HLO was renamed Health Literacy Online: A Guide for Simplifying the User Experience. The update included a website re-design and checklist to convey all of its design recommendations. The HLO Checklist [9] contains five categories that reflect chapters of the resource:

1. Write Actionable Content

2. Display Content Clearly on the Page

3. Organize Content and Simplify Navigation

4. Engage Users, and 
5. Test your Site with Users With Limited Health Literacy

The introduction of a checklist created an easier approach for using HLO, not only as a design resource, but as a consumer HIS assessment tool as well. Researchers have used the HLO Checklist as an evaluation framework to identify eHealth literacy and usability issues in consumer HISs (e.g., [10], [13]).

\section{Evidenced-Based Heuristics for eHealth Literacy and Usability}

While using the guidelines based on the original HLO site [14], researchers identified shortcomings and missing empirical evidence that could be included to bolster these guidelines. To that end, we developed a more comprehensive set of heuristics based on content from original HLO site, but complemented with findings from other studies on eHealth literacy and usability [15]. The set Evidence-Based Heuristics includes 8 core and 3 optional heuristics:

1. Immediately Inform Users of Purpose and Engage Users, Avoid Registration

2. Use Complementary Interaction Methods

3. Leverage Interactivity

4. Provide Accurate, Colloquial, Comprehensive, Succinct Content

5. Provide Tailored, Flexible, Layered Content

6. Use Visuals to Complement Text, But Avoid Tables

7. Simplistic, Consistent Navigation

8. Simplistic, Consistent Displays

9. (Optional) Clear and Comprehensive Communication of Risks

10. (Optional) Clear Depiction of Monitoring Data and/or Test Results

11. (Optional) Considerations for Mobile Devices

We, amongst others, have found these Evidence-Based Heuristics to be a valuable method for assessing consumer HISs and identifying opportunities to improve the UX and make the content more understandable (e.g., $[16,17])$.

\section{Comparison}

To compare these two tools (i.e., the HLO Checklist and the Evidence-Based Heuristics), the authors relied on their previous experience and other researcher-reported experiences with the tools. For example, we previously used the Evidence-Based Heuristics to evaluate a blood pressure monitoring app revealing several problems in both eHealth literacy and usability (e.g., lack of alert for serious health concern) [15]. Recently, we used the HLO Checklist to evaluate an app to convert online lab results into citizenfriendly formats, again identifying several usability and eHealth literacy problems (e.g., extensive medical jargon) [in press]. Additionally, another group of researchers used both methods [14]. In their subsequent study, the researchers [18] reported that the HLO checklist "was easier to use and returned more specific results than the second heuristic set" (i.e., Evidence-Based Heuristics), with two exceptions which they integrated into their inspection checklist. We categorized these findings into strengths and weaknesses for each tool and examined them for commonalities. Therefore, having first-hand experience using both tools and insight into other researchers' experiences from 
published materials allowed us to identify the unique and common strengths and weaknesses of both tools (see Figure 1).

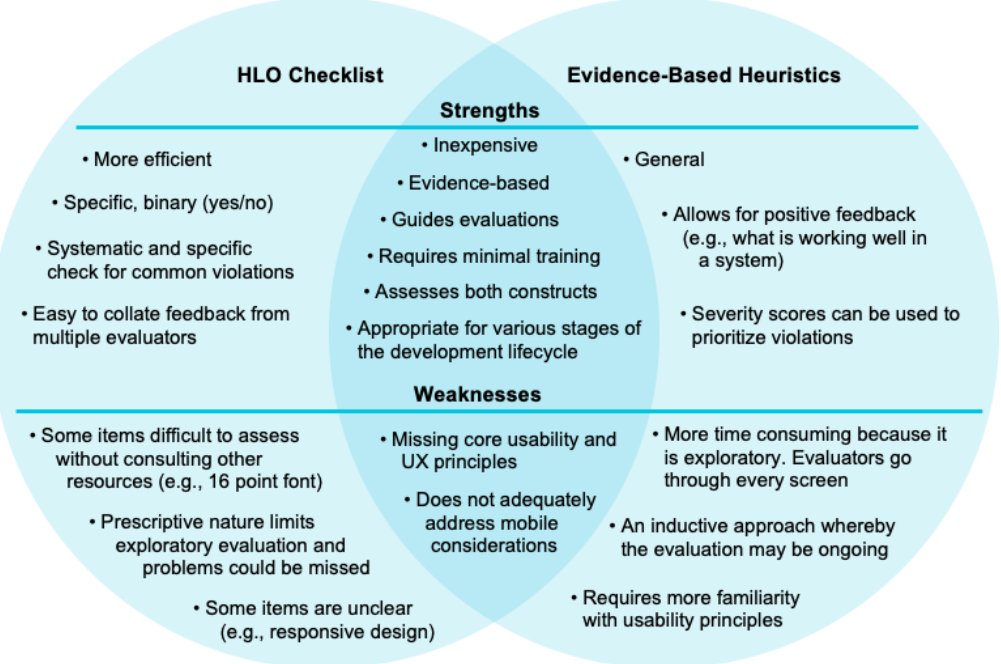

Figure 1. Comparison of the strengths and weaknesses of the two inspection methods.

\section{Discussion}

This study revealed that neither method available is optimal for evaluating UX and eHealth literacy demands of consumer HISs. The HLO checklist is easier for those less familiar with the domains of eHealth Literacy and usability to employ because it is more specific and binary. However, relying solely on the HLO checklist would result in evaluations largely ignoring the importance of UX. Yet even the Evidence-Based Heuristics do not provide a complete UX assessment. Having a comprehensive checklist tool could improve uptake and make it easier for many stakeholders (i.e., citizens, health care providers, and app developers) to conduct their own evaluations. Therefore, future research is warranted to develop a checklist tool that can assess eHealth literacy and UX in conjunction. The goal would be to combine the strengths of both tools, while mitigating their weaknesses, requiring the following steps:

1. To amplify the UX component, a comprehensive set of heuristics should be established to include heuristics from a general usability set (e.g., [19]) and others specific to mobile and UX (e.g., perceptions, responses);

2. This resultant set of heuristics should then be converted into checklist items.

3. These items should be added to the HLO checklist to create an easy-to-use, comprehensive tool for both eHealth literacy and UX that could be validated.

Given their relative ease of use, adopting checklist assessment tools may also be warranted for inspecting clinical HISs.

A limitation of this study was that the lead author [HM] developed the EvidencedBased Heuristics. To reduce this influence on the comparison, the researchers used bracketing to make any biases explicit. For example, we acknowledge that our tool, created seven years ago, likely requires updating and may be more challenging for non- 
experts to use. The second author [JG] was not involved in the creation of the tool and was able to provide a neutral lens. However, we believe that having used both tools on multiple occasions, provided for a more fulsome critique.

In an increasingly digital world, where the health care landscape is rapidly transforming, citizens deserve consumer HISs that provide excellent UX and place appropriate demands on eHealth literacy. Moreover, improved UX could even serve as a mechanism to improving eHealth literacy and ultimately health outcomes by increasing engagement, education, and self-management. Thus, it is important to develop efficient, easy to use tools for assessing both of these constructs simultaneously in consumer HISs.

\section{References}

[1] Conducted by TNS Political \& Social at the request of. European citizens' digital health literacy: report. [Internet]. LU: Publications Office; 2014. Available at: https://data.europa.eu/doi/10.2759/86596, Accessed 2021 Mar 7.

[2] IQVIA Institute for Data Science. The Growing Value of Digital Health: Evidence and Impact on Human Health and the Healthcare System [Internet]. 2017. p. 76. Available at: https://www.iqvia.com/$/ \mathrm{media} /$ iqvia/pdfs/institute-reports/the-growing-value-of-digital-health.pdf? =1611945725228.

[3] Kushniruk A, Kaipio J, Nieminen M, et al. Comparing approaches to measuring the adoption and usability of electronic health records: lessons learned from Canada, Denmark and Finland. Stud Health Technol Inform. 2013;192:367-371.

[4] ISO 9241-11:2018(en), Ergonomics of human-system interaction - Part 11: Usability: Definitions and concepts [Internet]. Available at: https://www.iso.org/obp/ui/\#iso:std:iso:9241:-11:ed-2:v1:en, Accessed 2021 Jan 29.

[5] Norman CD, Skinner HA. eHealth Literacy: Essential Skills for Consumer Health in a Networked World. J Med Internet Res. 2006;8:e9.

[6] Monkman H, Kushniruk AW. The Consumer Health Information System Adoption Model. Stud Health Technol Inform. 2015;218:26-31.

[7] Segall N, Saville JG, L'Engle P, et al. Usability evaluation of a personal health record. AMIA Annu Symp Proc AMIA Symp. 2011;2011:1233-1242.

[8] Monkman H, Kushniruk A. Applying usability methods to identify health literacy issues: an example using a Personal Health Record. Stud Health Technol Inform. 2013;183:179-185.

[9] Checklist - Health Literacy Online | health.gov [Internet]. Available at: https://health.gov/healthliteracyonline/checklist/. Accessed 2021 Jan 2.

[10] Monkman H, Schmidt T, Nøhr C. Online Medication Information for Citizens: A Comparison of Demands on eHealth Literacy. Stud Health Technol Inform. 2020;270:1026-1030.

[11] Nielsen J. Usability inspection methods. Conf Companion Hum Factors Comput Syst - CHI 94 [Internet]. Boston, Massachusetts, United States: ACM Press; 1994. p. 413-414. Available at: http://portal.acm.org/citation.cfm?doid=259963.260531. Accessed cited 2021 Jan 31.

[12] Hou S-I. Health Literacy Online: A Guide to Writing and Designing Easy-to-Use Health Web Sites. Health Promot Pract. 2012;13:577-580.

[13] Walsh L, Hemsley B, Allan M, et al. The E-health Literacy Demands of Australia's My Health Record: A Heuristic Evaluation of Usability. Perspect Health Inf Manag. 2017;14:1f.

[14] Monkman H, Kushniruk A. A health literacy and usability heuristic evaluation of a mobile consumer health application. Stud Health Technol Inform. 2013;192:724-728.

[15] Monkman H, Griffith J, Kushniruk AW. Evidence-based Heuristics for Evaluating Demands on eHealth Literacy and Usability in a Mobile Consumer Health Application. Stud Health Technol Inform. 2015;216:358-362.

[16] Coughlin SS, Besenyi GM, Bowen D, et al. Development of the Physical activity and Your Nutrition for Cancer (PYNC) smartphone app for preventing breast cancer in women. mHealth. 2017;3:5-5.

[17] Monkman H, Mavriplis C, Griffith J. Putting Guidelines in the Hands of Patients: A Heuristic Evaluation of a Consumer Mobile Application. Stud Health Technol Inform. 2019;257:314-318.

[18] Walsh L, Hemsley B, Allan M, et al. Assessing the information quality and usability of My Health Record within a health literacy framework: What's changed since 2016? Health Inf Manag J Health Inf Manag Assoc Aust. 2021;50:13-25.

[19] Nielsen J. 10 Usability Heuristics for User Interface Design [Internet]. Nielsen Norman Group. Available at: https://www.nngroup.com/articles/ten-usability-heuristics/. Accessed 2021 Jan 31. 